\title{
“A new wiki way?” - An experimental study of collaborative knowledge building scripts
}

\author{
Sven Heimbuch ${ }^{1}$, Kerstin Uhde ${ }^{1}$, and Daniel Bodemer ${ }^{1}$ \\ ${ }^{1}$ University of Duisburg-Essen, Germany
}

Corresponding author: Sven Heimbuch

Email address: sven.heimbuch@uni-due.de

\section{ABSTRACT}

One of Wikipedia's main guiding principles reads as follows: Be Bold. From Wikipedia's early days on users were encouraged to share their knowledge at any convenient time without major restrictions by either adding new content or editing the encyclopaedia's existing content in the sense of knowledge building in collaborative writing systems. In order to ensure that authors and editors of articles successfully collaborate on shared knowledge artifacts, rules and guidelines have been implemented into the community closely related to the "Be Bold" principle. One of these guidelines is the so-called Bold, Revert, Discuss (BRD) Cycle that describes a workflow corresponding to a collaboration script on how individuals in an environment like Wikipedia should interact with each other to jointly create and manage articles. This cycle was primarily implemented to attract masses of new authors and editors to the community by lowering the bar for participation. From today's point of view it is debatable if this procedure should be further promoted or if alternative methods as the in this study proposed script Discuss, Deliberate, Revise (DDR) prove themselves as more effective for knowledge building with a special focus on socio-cognitive conflicts in wikis. We expect that students collaboratively working together using the DDR alternative script will produce qualitatively higher articles and will perform better in a knowledge test about the study's topic.

\section{INTRODUCTION}

Collaborative writing systems such as Wikipedia provide an open environment for collaborative learning by facilitating knowledge building processes in individuals and in the wiki itself as social system. Scardamalia and Bereiter (1994) have defined knowledge building as the creation of knowledge as a social product. In recent years research has been conducted on how learning processes and knowledge building can be supported by measures of computer-supported collaboration. In this specific area of knowledge building in wikis significant research was inspired by Piaget's constructivist school of thought (Cress and Kimmerle, 2008). According to the resulting Co-Evolution Model, analogous processes of internalisation and externalisation can be found in an individual's cognitive system as well as on a wiki's system level. By mutually influencing each other's system, at every level manifold possibilities for socio-cognitive conflicts can arise if either one cognitive system's knowledge base dissents the social system or vice versa.

In collaborative learning scenarios making use of socio-cognitive conflicts plays an important role. Bell et al. (1985) examined such conflicts that emerged if an individual is confronted with a different perspective contradictory to its own cognitive representation. Such conflicts lead to reorganisation and reconstruction of cognitive processes and furthermore to a success in learning, if the achievement of a consensus is required or desired. Similar aims have been followed by research on Constructive Controversy (Johnson et al., 2000), a script that structures decision-making and learning processes. Learners benefit from conflicts that have been systematically used and developed with opposing positions in mind. As a result, higher-quality argumentation, more complex formulation of con- 
cepts and more critical examinations of discussed topics can emerge.

Furthermore, in the area of computer-supported collaborative learning a number of different collaboration scripts for have been analysed and compared with each other (Dillenbourg, 2002). As a result, the meaning of social interaction was emphasized as a core element for collaborative learning: "From the designer's viewpoint, a script remains within the 'collaborative learning' philosophy if the script design rationale calls upon social interaction as core learning mechanism, not simply as an add-on to individual activities" (p. 28). In order to further examine collaboration scripts especially in wiki-based environments, Wichmann and Rummel (2013) were interested in the effectiveness of a specific script that aimed at improving students' revision behaviour by prompting them to distribute their prior knowledge. The control group that worked on the article without any script wrote shorter articles, revised the articles less frequent and produced less coherent articles compared to the scripting condition. In addition to that, a positive correlation between the number of revisions and the coherency of the article has been observed. According to Papadopoulos et al. (2013) who examined the degrees of freedom script should provide, the level of coercion has an impact on the learning success. Higher coercion in scripted collaboration led to better learning outcomes, achieved by being encouraged to deeper elaborate the learning materials and by lowering extraneous cognitive load.

On the basis of the previously discussed research, in the following study we are mainly interested in the research question, if learners in collaborative writing scenarios benefit from a collaboration script that is primarily focussed on discussing the implementation of knowledge artefacts before an edit is conducted. This proposed Discuss, Deliberate, Revise (DDR) script will be contrasted against the Bold, Revert, Discuss (BRD) cycle that was originally proposed by Wikipedia with a focus on encouraging editors and authors to quickly implement or edit knowledge artefacts. Further questions of interest are, if complying with our proposed alternative script will lead to higher quality articles and to what degree will knowledge building processes be influenced by individual differences in the cognitive variable Need for Cognitive Closure (Kruglanski and Webster, 1996).
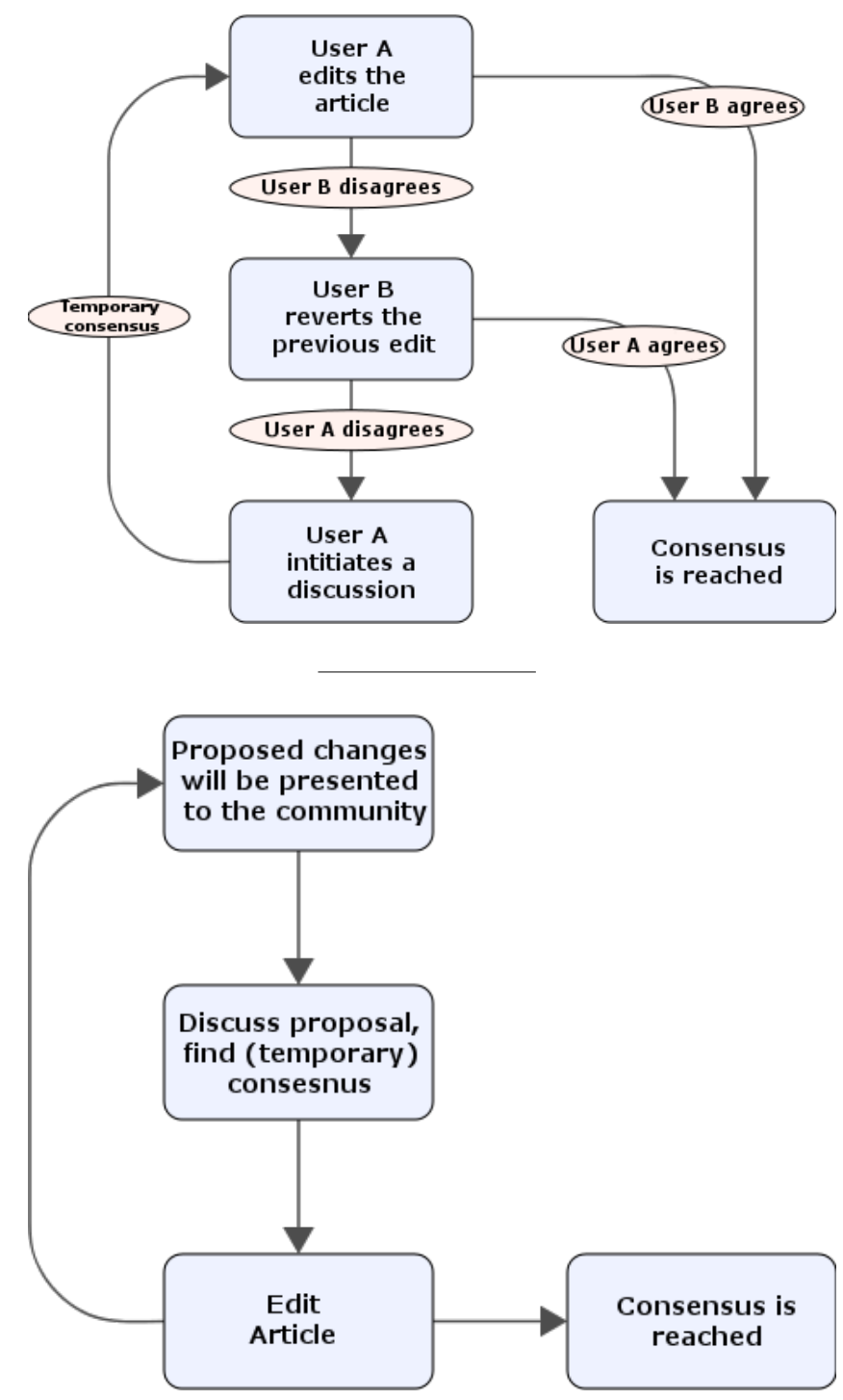

Figure 1. Illustrations of the respective article editors with BRD script (top) and DDR script (bottom).

\section{METHODS}

In order to answer the interested research questions, an experimental study is currently conducted in a controlled laboratory setting with approximately $N$ $=60$ students $(N=30$ dyads $)$ at the end of the study. Two different collaboration scripts will be imple- 


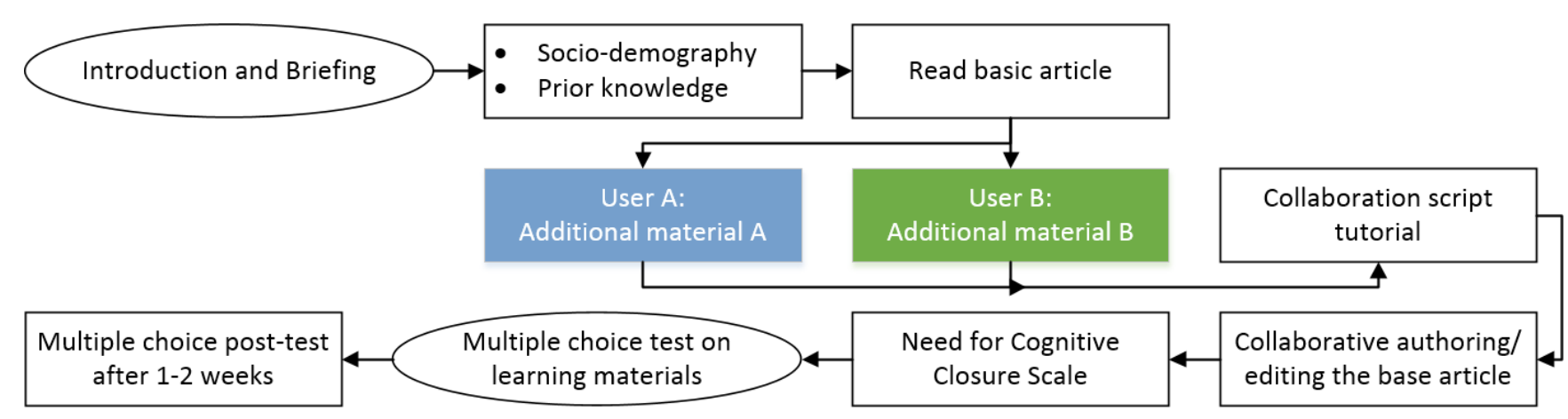

Figure 2. Study procedure for both experimental conditions.

mented and compared against each other as the independent variable which will be randomly assigned to each dyad. Figure 1 illustrates the condition's article editors and visual representations of the corresponding collaboration scripts. The BRD script corresponds to the original workflow proposed by Wikipedia, whereas the alternative DDR script is self-developed inspired by previous research on coordinated work in wikis where higher level of coercion to discuss before editing was enacted.

The study's contents are on a pirate captain for whom contradictory information on several aspects of his life exists. At first, both participants in a dyad will have to read the same basic article to establish a common ground. Followed by that, opposing historical facts (learning material A or B) will be presented to either learning partner, in order to facilitate socio-cognitive conflicts to arise. The common task will be to collaboratively author the basic article by editing existing paragraphs or adding entirely new artefacts to the article. After the collaborative writing task both participants will have to answer a multiple choice test that can only be fully solved if contents of both additional learning materials $\mathrm{A}$ and $B$ have been made known to each partner. The complete study procedure is illustrated in Figure 2. The currently conducted study is scheduled to be finished until late September.

\section{OUTLOOK}

For our planned statistical analyses, the recorded data on individual learning success measured by two multiple choice tests at two different dates about the study's content will be processed and evaluated. Furthermore, qualitative content analyses on the final written texts of each dyad in both experimental conditions will be conducted. Finally, the influence of an individual's Need for Cognitive Closure on the resulting article and the performance in both multiple choice tests will be examined in detail. We expect the proposed alternative script (DDR) group to outperform the control (BRD) group on the quantitative as well as on the qualitative variables.

\section{REFERENCES}

Bell, N., Grossen, M., and Perret-Clermont, A.-N. (1985). Sociocognitive conflict and intellectual growth. New Directions for Child and Adolescent Development, 1985(29):41-54.

Cress, U. and Kimmerle, J. (2008). A systemic and cognitive view on collaborative knowledge building with wikis. International Journal of Computer-Supported Collaborative Learning, 3(2):105-122.

Dillenbourg, P. (2002). Over-scripting CSCL: The risks of blending collaborative learning with instructional design. Three worlds of CSCL. Can we support CSCL?, pages 61-91.

Johnson, D. W., Johnson, R. T., and Smith, K. A. (2000). Constructive controversy: The educative power of intellectual conflict. Change: The Magazine of Higher Learning, 32(1):28-37.

Kruglanski, A. W. and Webster, D. M. (1996). Motivated closing of the mind: "Seizing" and "freezing.". Psychological Review, 103(2):263-283. 
Papadopoulos, P., Demetriadis, S., and Weinberger, A. (2013). 'Make it explicit!': Improving collaboration through increase of script coercion. Journal of Computer Assisted Learning, 29(4):383398.

Scardamalia, M. and Bereiter, C. (1994). Computer support for knowledge-building communities. Journal of the Learning Sciences, 3(3):265283.

Wichmann, A. and Rummel, N. (2013). Improving revision in wiki-based writing: Coordination pays off. Computers \& Education, 62:262-270. 